\title{
Fluctuation-Dominated Kinetics under Stirring
}

\author{
R. Reigada, ${ }^{1}$ F. Sagués, ${ }^{1}$ I. M. Sokolov, ${ }^{2,3}$ J. M. Sancho, ${ }^{4}$ and A. Blumen ${ }^{2}$ \\ ${ }^{1}$ Departament de Química Física, Universitat de Barcelona, Diagonal 647, Barcelona 08028, Spain \\ ${ }^{2}$ Theoretische Polymerphysik, Universität Freiburg, Rheinstrasse 12, D-79104 Freiburg i. Br., Germany \\ ${ }^{3}$ P. N. Lebedev Physical Institute of the Academy of Sciences of Russia, Leninski Prospect 53, Moscow 117924, Russia \\ ${ }^{4}$ Departament d'Estructura i Constituents de la Matèria, Universitat de Barcelona, Diagonal 647, Barcelona 08028, Spain
}

(Received 9 May 1996)

\begin{abstract}
We investigate the effects of stirring on the kinetics of the $A+B \rightarrow 0$ reaction under stoichiometrical conditions in $2 \mathrm{D}$. We consider both a steady eddy-lattice flow and a random flow mimicking turbulence. In both situations complex decay patterns are detected. Only an intermediate stage of the reaction is dominated by mixing. The long-time behavior shows fluctuation-dominated kinetics, $c(t) \propto t^{-1 / 2}$, governed by effective diffusion. For the case of an eddy-lattice flow a very slow intermediate regime emerges, $c(t) \propto t^{-1 / 4}$, which is associated with the closed topology of flow lines. [S0031-9007(96)02254-5]
\end{abstract}

PACS numbers: 82.20.- $\mathrm{w}, 05.40 .+\mathrm{j}, 47.70 .-\mathrm{n}$

Chemical processes often depend on stirring to homogenize the reactants [1-3]. In fact, due to the sensitivity of the reactions on the mixing procedure, qualitatively different kinetic patterns show up [3]. This is especially important for reactions leading to segregation. In the present Letter we consider the kinetics of the $A+B \rightarrow 0$ reaction under stoichiometrical conditions, taking place under twodimensional model flows.

As is by now well known, under diffusion the average concentration $c(t)$ of (stoichiometric) reactants follows $d$ dimensions a power law $c(t) \sim(D t)^{-d / 4}$ (where $d \leq$ 4) [4-10], which is slower than the classical kinetics form $c(t) \propto t^{-1}$. The classical form is obeyed when the concentrations are homogeneous at all times, which can be achieved through efficient procedures such as mixing by dilatational flow [11], tossing [12], or unbounded shear flow [13-15]. In general realistic mixing flows, especially in two dimensions, are less effective [1]. We display this here by considering two flow patterns, one related to Rayleigh-Bénard convection [16] (a steady twodimensional lattice of eddies), the other one being a random flow, which mimics turbulent stirring. A variety of - rather unexpected-kinetic regimes appears.

We describe the system in terms of reaction-diffusionadvection equations for the local densities $c_{A, B}(\mathbf{r}, t)$, having

$$
\frac{\partial c_{A}}{\partial t}+\mathbf{v} \cdot \nabla c_{A}=D \Delta c_{A}-k c_{A} c_{B}
$$

and

$$
\frac{\partial c_{B}}{\partial t}+\mathbf{v} \cdot \nabla c_{B}=D \Delta c_{B}-k c_{A} c_{B} .
$$

This is the standard approximate scheme [17-19] where $k$ denotes the local reaction rate coefficient and $D$ the molecular diffusivity. The scheme is qualitatively correct in higher dimensions for the reactants passively transported by the flow. The continuous-medium approach, Eqs. (1) and (2), is valid then on length scales much larger than the mean interparticle distance and supposes that the velocity does not change considerably on these scales. In our numerical work here, which parallels Ref. [9], we use $D=D_{A}=D_{B}=0.1$ and $k=10$ and monitor $c(t)$, the mean concentration $c(t)=\left\langle c_{A}(\mathbf{r}, t)\right\rangle=\left\langle c_{B}(\mathbf{r}, t)\right\rangle$. The initial conditions correspond to random distributions of reactants with $c(0)=1$. The incompressible velocity field $\mathbf{v}$ is given through the stream function $\eta(x, y)$ via

$$
\mathbf{v}(\mathbf{r})=(-\partial \eta / \partial y, \partial \eta / \partial x) .
$$

The stationary eddy-lattice flow is modeled using

$$
\eta(x, y)=\left(2 L u_{0} / n \pi\right) \cos (n \pi x / L) \cos (n \pi y / L),
$$

while for the "synthetic" time-dependent turbulent flow we take $\eta(x, y, t)$ to be a Gaussian random process in space and time, whose correlation functions are chosen to reproduce closely the realistic properties of turbulent flows. This pragmatic point of view allows us to display the role of generic effects of turbulence on chemical reactions. In what follows a Kraichnan's energy spectrum $E(k) \propto$ $k^{3} \exp \left(-k^{2} / k_{0}^{2}\right)$ is used. The random function $\eta(x, y, t)$ is built up from its Fourier harmonics, each one of them satisfying its own Langevin equation. A detailed account of the numerical procedure is given in Refs. [20-22].

Figure 1 displays on double-logarithmic scales the $c(t)$ behavior for the eddy-lattice flow. The parameters in Fig. 1(a) are chosen such as to include (however fleetingly) all possible decay regimes. Each of these regimes can be shown in more detail through a judicious choice of parameters in each particular case; as, e.g., in Figs. 1(b) and 1(c). The spatial organization of reactants is shown in Fig. 2. After a fluctuation-dominated initial stage, Fig. 2, upper left, the reaction enters a regime which is determined by mixing along flow lines. For intense flows this regime tends to follow a $c \propto t^{-1}$ law. 

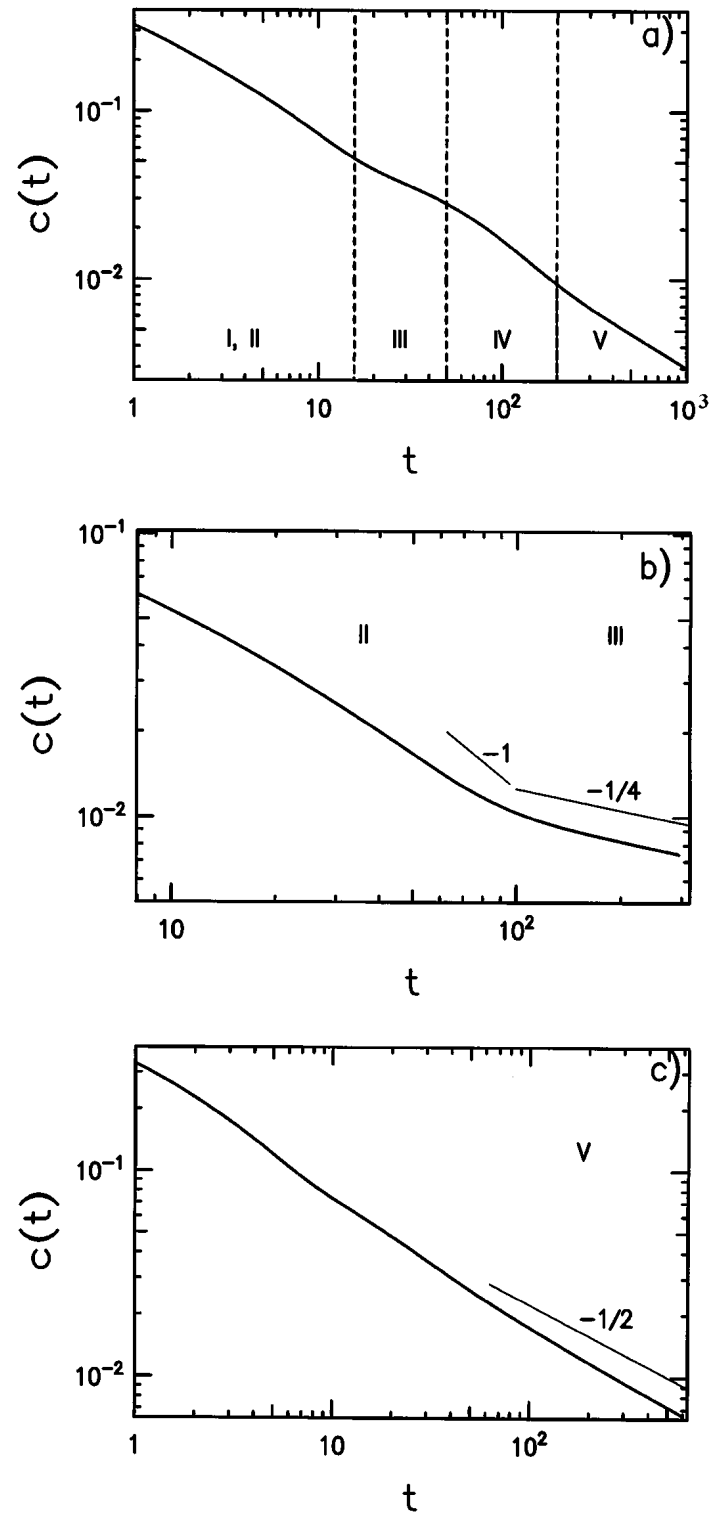

FIG. 1. (a) Plot of $c(t)$ vs $t$ for the eddy-lattice flow with $u_{0}=2, L=300$, and $n=30$, obtained as an average over three realizations. The roman figures I and II denote the initial stages of the reaction and the mixing along the flow lines, whereas III corresponds to the slow diffusion across the flow lines, after which the transient regime IV and the asymptotic $c \propto t^{-1 / 2}$-regime $\mathrm{V}$ follow. (b) Plot of $c(t)$ for $u_{0}=10, L=150$, and $n=2$ to render the crossover from II to III ( $c \propto t^{-1 / 4}$-regime) clear. (c) Plot of $c(t)$ for $u_{0}=2$, $L=300$, and $n=60$ to exhibit regime $\mathrm{V}, c \propto t^{-1 / 2}$. Note the different scales.

At longer times a crossover towards a much slower kinetics, of one-dimensional type, $c \propto t^{-1 / 4}$ takes place, as can be clearly seen in Fig. 1(b). The slower kinetics is associated with the equilibration of concentrations across closed flow lines, e.g., the concentric rings pattern of Fig. 2, upper right. After this stage the reaction inside each individual eddy has practically finished; one is left with a quasiperiodic structure, Fig. 2, middle left, which
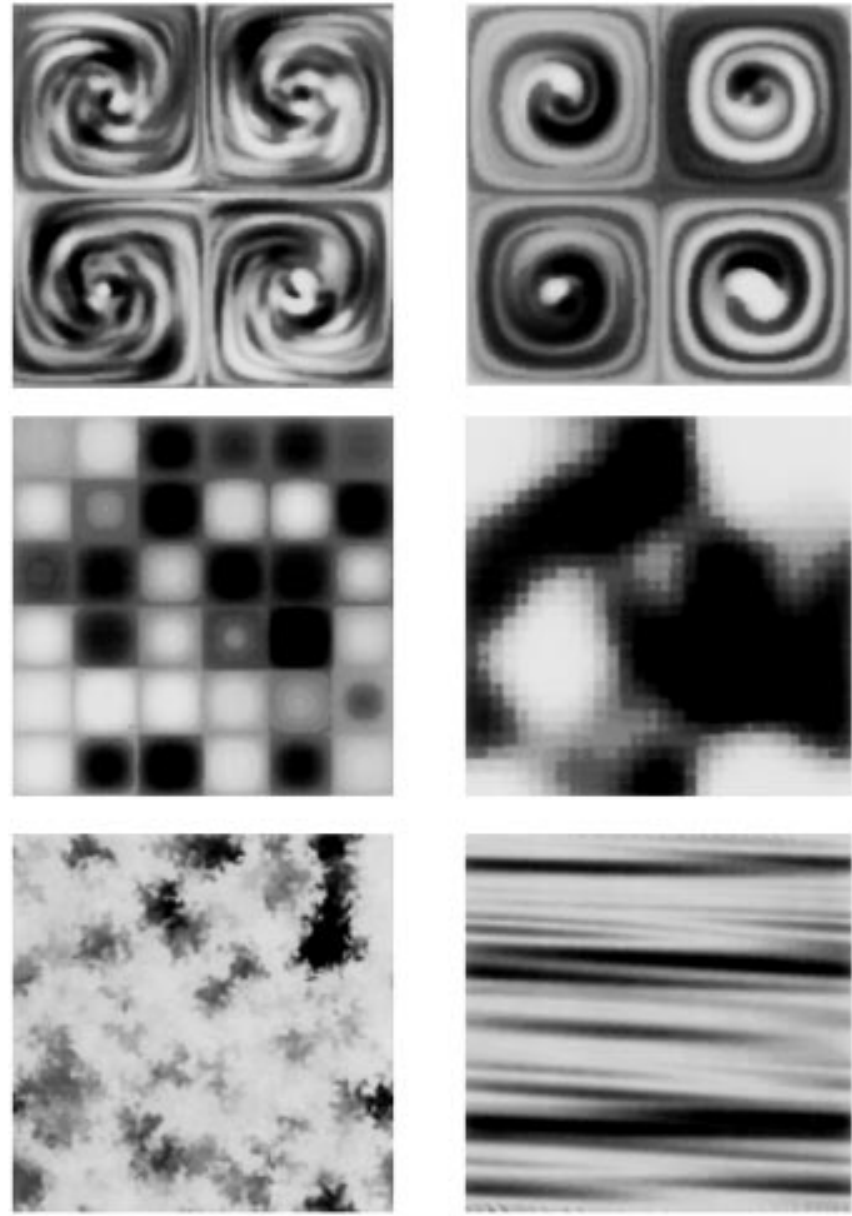

FIG. 2. Mosaic of patterns showing the spatial organization of reactants, where the grey scale represents $c_{A}$ and $c_{B}$; the maximum of $A$ is white and of $B$ black. Upper left: snapshot at $t=24$ of a simulation run with $u_{0}=10, L=150$, and $n=2$ (regime II). Upper right: as before but now at $t=60$ (regime III). Middle left: snapshot at $t=300$ for $u_{0}=10$, $L=150$, and $n=6$, showing regime IV. Middle right: snapshot at $t=800$ of a simulation run with the parameters of Fig. 1(a) (regime V). Lower left: a situation under turbulent flow at $t=90$ and with the parameters of Fig. 3. Lower right: spatial organization of reactants in a cylindrical shear flow with $\alpha=0.16$ and $L=150$ at $t=100$.

leads to a fast (quasiexponential) decay. Later the spatial structure evolves as in Fig. 2, middle right, and the decay is $c \propto t^{-1 / 2}$, i.e., two-dimensional fluctuation-dominated; see Fig. 1(c).

The turbulent flow shows three consecutive types of behavior (Fig. 3). After an initial stage, the kinetics follows the classical $c(t) \propto t^{-1}$ law due to mixing. Later on the decay crosses over to a fluctuation-dominated regime, $c(t) \propto t^{-1 / 2}$, governed by an effective (turbulent) diffusion. The spatial organization of reactants for this case is shown in Fig. 2, lower left. For the sake of comparison Fig. 3 also shows $c(t)$ for two nonadvected systems $(\mathbf{v}=0)$, one with a diffusion coefficient equal to $D$ and the other with an effective coefficient $D^{*}$, due 


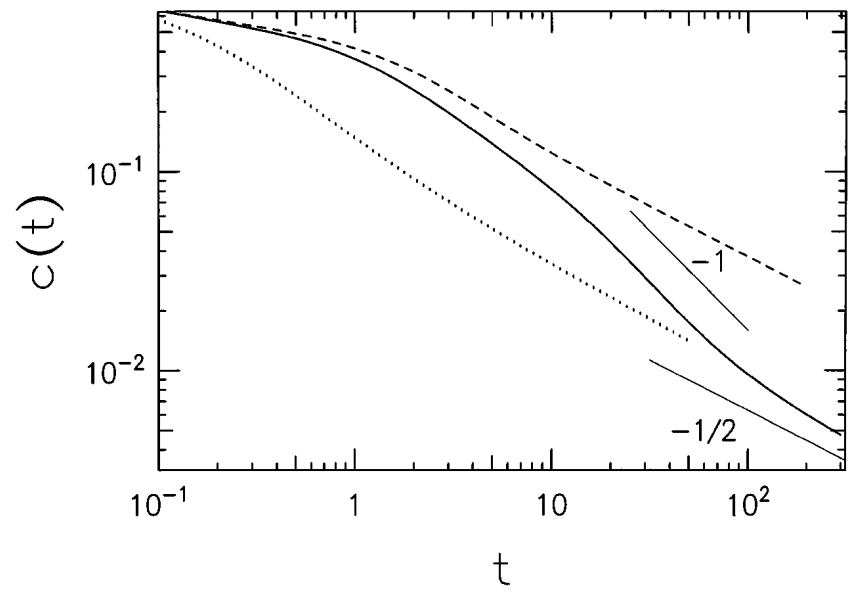

FIG. 3. Same as Fig. 1 but now for synthetic turbulent flow in a system with $L=300$. The flow has the velocity intensity $u_{0}^{2}=1.243$, a correlation length of $l_{0}=1.7$, and a correlation time $t_{0}=2$. The dashed line corresponds to a nonadvected reactive system with $D=0.1$, the dotted one to a nonadvected reactive system with $D^{*}=1.529$. All the results presented are averages over five realizations. The straight lines have slopes -1 and $-1 / 2$, respectively.

to turbulent diffusion (calculated for the advected scalar $[21,22])$. The mixing stage interpolates between the shorttime and the long-time regimes, which are governed by $D$ and $D^{*}$, respectively.

Common to both types of flow are the following three regimes: an initial diffusion-controlled regime (small scale clustering), a fast ("classical") regime due to mixing at intermediate scales, and a long-time, again diffusioncontrolled asymptotic behavior (large scale clustering).

We return now to the theoretical explanation of the intermediate slowing down in the case of an eddy lattice. As already mentioned, this slowing down of the reaction (which leads to the $t^{-1 / 4}$ decay pattern) is related to the closed geometry of the flow lines. Thus the concentration fluctuations along the flow lines are smoothed out by mixing, while in the direction perpendicular to the flow lines the equilibration is due only to the much slower process of molecular diffusion. Since for eddy-lattice flow no exact solution of the diffusion equation is known, we look for simpler flows which display the property of interest, namely, closed flow lines. Such a model is provided by the shear flow on a cylindrical surface, with the axis of the cylinder pointing in the $y$ direction and $x$ being the coordinate along the circular cross section $(0 \leq x<L)$. The flow is $\mathbf{v}=(\alpha y, 0)$. The numerical results for this flow are given in Fig. 4.

From Eqs. (1) and (2) the difference in local concentrations, $q(\mathbf{r}, t) \equiv c_{A}(\mathbf{r}, t)-c_{B}(\mathbf{r}, t)$, is governed by the equation $\frac{\partial q}{\partial t}+\mathbf{v} \cdot \nabla q=D \Delta q$ [3]. The solution of this equation can be expressed through its Green's function $G\left(\mathbf{r}, \mathbf{r}^{\prime}, t\right)$, which for the flow considered can be built up, using a reflection argument, along the lines of Ref. [23]. The calculations parallel those of Refs. $[3,9,10]$ and lead

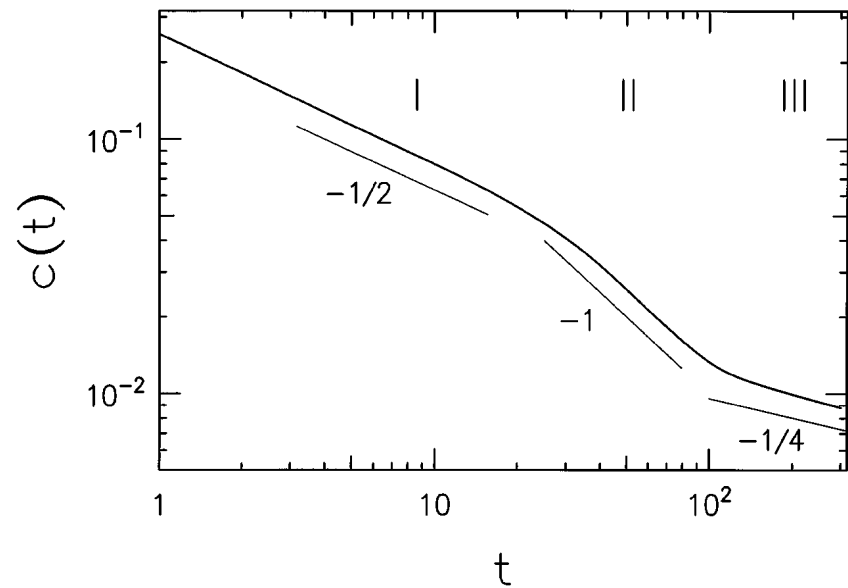

FIG. 4. Same as Fig. 1 but now for shear flow on the surface of a cylinder with $\alpha=0.16$ and $L=150$. The slopes shown correspond to stages I, II, and III of Fig. 1. The results are averages over five realizations.

$$
\begin{aligned}
& \text { for }\left\langle q^{2}(t)\right\rangle \text { to } \\
& \qquad \begin{aligned}
\left\langle q^{2}(t)\right\rangle= & \frac{2 c(0)}{\pi L} \sqrt{\frac{\pi}{2 D t}} \sum_{n=0}^{\infty} \\
& \times \exp \left[-2 D t\left(1+\frac{\alpha^{2} t^{2}}{12}\right) \frac{\pi^{2}}{L^{2}} n^{2}\right] .
\end{aligned}
\end{aligned}
$$

Moreover, for fast reactions

$$
c(t)=\langle|q(\mathbf{r}, t)|\rangle / 2 \propto\left\langle q^{2}(t)\right\rangle^{1 / 2}
$$

holds. Equations (5) and (6) reproduce all three regimes seen in simulations and displayed in Fig. 4. For short times, $t<\left(6 L^{2} / \pi^{2} D \alpha^{2}\right)^{1 / 3}$, one can replace the summation in Eq. (5) by an integration, obtaining thus $\left\langle q^{2}(t)\right\rangle=$ $2 c(0) \sqrt{3} / \pi D t\left(\alpha^{2} t^{2}+12\right)^{1 / 2}$. Using this form one has that for very short times, $t<\alpha^{-1}$, the decay follows the two-dimensional diffusion-controlled kinetic pattern $c(t) \propto t^{-1 / 2}$, while for $t>\alpha^{-1}$ it follows the classical $c(t) \propto 1 / t$ behavior. For $t>\left(6 L^{2} / \pi^{2} D \alpha^{2}\right)^{1 / 3}$ only the first term in Eq. (5) is relevant, so that $c(t) \propto\left\langle q^{2}(t)\right\rangle^{1 / 2} \propto$ $t^{-1 / 4}$ holds. This last regime is due to a one-dimensional diffusion across flow lines. These three regimes in Fig. 4 correspond to the stages I to III in Fig. 1.

In summary, in the case of time-independent eddylattice flows, the overall kinetics is complex, showing different power laws. Especially interesting are the mixing-dominated intermediate stages, which show first a quick $c \propto t^{-1}$ decay and then a slow $c \propto t^{-1 / 4}$ decay. For reactions in a random flow which models turbulent stirring, three stages are seen: The mixing stage interpolates between the short-time behavior governed by the molecular diffusion coefficient $D$ and the long-time behavior, determined by an effective diffusion coefficient $D^{*}$. For both flows considered here, stirring does not imply mixing on very large scales, and is incapable to overcome segregation; the long-time behavior is hence diffusion controlled. 
The authors acknowledge discussions with Professor F.J. Muzzio and the support of the DFG, of the Fonds der Chemischen Industrie, of an "ACCIONES INTEGRADAS"-DAAD grant, of EC Grant No. CHRXCT93-0354, of the Comissionat per Universitats i Recerca de la Generalitat de Catalunya, and of the Dirección General de Investigación Científica y Tecnológica (DGICYT) Spain, under Projects No. PB93-759 and No. PB93-769.

[1] J. M. Ottino, The Kinematics of Mixing: Stretching, Chaos and Transport (Cambridge Univ. Press, Cambridge, 1989).

[2] S. Middleman, Fundamentals of Polymer Processing (McGraw-Hill, New York, 1977).

[3] I. M. Sokolov and A. Blumen, Int. J. Mod. Phys. B 5, 3127 (1991).

[4] A. A. Ovchinnikov and Ya. B. Zel'dovich, Chem. Phys. 28, 215 (1978).

[5] D. Toussaint and F. Wilczsek, J. Chem. Phys. 78, 2642 (1983).

[6] K. Kang and S. Redner, Phys. Rev. Lett. 52, 955 (1984); Phys. Rev. A 32, 435 (1985).

[7] I. M. Sokolov, JETP Lett. 44, 67 (1986).

[8] F. Leyvraz and S. Redner, Phys. Rev. Lett. 66, 2168 (1991).

[9] P. Argyrakis, R. Kopelman, and K. Lindenberg, Chem. Phys. 177, 693 (1993).

[10] R. Reigada, F. Sagués, I. M. Sokolov, J. M. Sancho, and A. Blumen, Phys. Rev. E 53, 3167 (1996).
[11] I. M. Sokolov and A. Blumen, Phys. Rev. Lett. 66, 1942 (1991); J. Phys. A 24, 3687 (1991).

[12] P. Argyrakis and R. Kopelman, J. Phys. Chem. 91, 2699 (1987); J. Phys. Chem. 93, 225 (1989).

[13] I. M. Sokolov and A. Blumen, in Synthesis, Characterization, and Theory of Polymeric Networks and Gels, edited by S.M. Aharony (Plenum, New York, 1992), p. 53.

[14] C. R. Doering and W. Horsthemke, Phys. Lett. A 182, 227 (1993).

[15] M. J. Howard and G. T. Barkema, Phys. Rev. E 53, 5949 (1996).

[16] A. Crisanti, M. Falcioni, G. Paladin, and A. Vulpiani, Riv. Nuovo Cimento 14, No. 12, 1 (1991).

[17] V. N. Kuzovkov and E. A. Kotomin, Rep. Prog. Phys. 51, 1479 (1988).

[18] H. Schnörer, V. Kuzovkov, and A. Blumen, Phys. Rev. Lett. 63, 805 (1989).

[19] G. Zumofen, J. Klafter, and A. Blumen, Phys. Rev. A 44, 8390 (1991).

[20] A. Careta, F. Sagués, and J. M. Sancho, Phys. Rev. E 48, 2279 (1993).

[21] A. Marti, A. Careta, J. M. Sancho, and F. Sagués (to be published).

[22] A. Careta, F. Sagués, and J. M. Sancho, Phys. Fluids 6, 349 (1994).

[23] A. S. Monin and A. M. Yaglom, Statistical Fluid Mechanics: Mechanics of Turbulence (MIT Press, Cambridge, MA, 1979), Vol. 1, Chap. 9. 